\title{
Knockdown of Gastrin Promotes Apoptosis of Gastric Cancer Cells by Decreasing ROS Generation
}

\author{
Yan Liu $\left(\mathbb{D},{ }^{1}\right.$ Jihai Zhu $\mathbb{D}^{2},{ }^{2}$ Jun Liu $\mathbb{D}^{1},{ }^{1}$ Xueman Ma ${ }^{\mathbb{D}},{ }^{1}$ Jun Zhao ${ }^{\mathbb{D}},{ }^{1}$ and Zhanhai Su ${ }^{1}{ }^{1}$ \\ ${ }^{1}$ Immunology Department, School of Medicine, Qinghai University, Qinghai 810000, China \\ ${ }^{2}$ Cardiovascular Surgery Department, The Affiliated Hospital of Qinghai University, Qinghai 810000, China
}

Correspondence should be addressed to Zhanhai Su; suzhanhai123@126.com

Received 8 January 2021; Accepted 1 April 2021; Published 15 April 2021

Academic Editor: Saheem Ahmad

Copyright (C) 2021 Yan Liu et al. This is an open access article distributed under the Creative Commons Attribution License, which permits unrestricted use, distribution, and reproduction in any medium, provided the original work is properly cited.

\begin{abstract}
Overexpressed gastrin is reported to promote oncogenesis and development of gastric cancer by inhibiting apoptosis of cancer cells; however, the underlying mechanism remains unclear. Our study is aimed at revealing the mechanism underlying the effect of gastrin on apoptosis of gastric cancer cells. Gastrin-interfering cell line was constructed by stably transfecting gastrin-specific pshRNA plasmid to gastric cancer cell line BGC-823. Then, differentially expressed proteins between untreated BGC-823 and gastrin-interfering BGC-823 cell lines were detected by the iTRAQ technique. GO and KEGG analysis was used to analyze the differentially expressed genes that code these differentially expressed proteins. The Annexin V-FITC staining assay was used to detect gastric cancer cell apoptosis. The DCFH-DA fluorescent probe staining assay was used to measure intracellular ROS. Mitochondrial membrane potential was detected by flow cytometry. Western blot was used to analyze the mitochondria respiratory chain proteins and apoptosis-related proteins. A total of 107 differentially expressed proteins were identified by iTRAQ. GO and KEGG analysis showed that proteins coded by the corresponding differentially expressed genes were mainly enriched in the mitochondrial oxidative respiratory chain, and the expression of three proteins (COX17, COX5B, ATP5J) was upregulated. The three proteins with higher scores were verified by Western blot. The apoptosis rate of the gastrin knockdown cancer cell was significantly increased; meanwhile, gastrin knockdown leads to increase of membrane potential and decrease of intracellular ROS production. Additionally, Bax was significantly increased, whereas NF- $\kappa$ B-p65 and Bcl-2 were downregulated after knockdown of gastrin. Concomitantly, pretreatment with NAC reversed the effect of gastrin on the Bax and Bcl-2 expression. Gastrin promotes the production of ROS from mitochondria, activates NF- $\kappa \mathrm{B}$, and inhibits apoptosis via modulating the expression level of Bcl-2 and Bax.
\end{abstract}

\section{Introduction}

Gastrin is an important polypeptide hormone synthesized by gastroduodenal $\mathrm{G}$ cells and contributes to the regulation of a variety of gastrointestinal tract functions, including acid secretion, motility, and epithelial proliferation [1]. Despite the known role of gastrin as a normal gastrointestinal hormone, an increasing number of studies have shown its "dark side" [2-10]. Gastrin has been reported to be abnormally expressed in a variety of tumors, such as pancreatic cancer [3], colorectal cancer [4], lung cancer [5], and gastric cancer [6], and it can promote proliferation and metastasis of tumors [7], alter the immune cell signature in the tumor microenvironment [8], and inhibit cancer cell apoptosis $[9,10]$. To date, the role of gastrin in inhibiting apoptosis of gastric cancer cells has been fully elucidated $[11,12]$; however, the underlying mechanism remains to be further investigated.

Generally, mitochondria not only generate energy for the vital activities of eukaryotic cells but also participate in many biological processes, such as energy metabolism [13], apoptosis [14], ROS production [15], and calcium homeostasis $[16,17]$. Studies have shown that the abnormal expression of mitochondrial respiratory chain proteins is closely related to a variety of diseases, especially tumors $[18,19]$. In the 1930s, Otto Warburg suggested that "respiration damage" was a pivotal feature of cancer cells $[20,21]$. Subsequently, a large number of studies have 
shown a decrease of mitochondrial respiration and oxidative phosphorylation in cancer $[22,23]$. Today, the concept that mitochondrial dysfunction is one of the most prominent features of cancer cells has been widely accepted.

Mitochondria are the major source of cellular reactive oxygen species (ROS). In some cases, the inhibition of respiratory activity clearly leads to enhanced generation of ROS [22]. By acting both as mutagens and cellular mitogens, excessive ROS changes the cellular redox status and oxidative stress [23] and affects the activities of sensitive transcription factors to regulate the gene expression and promote cancer cell proliferation $[24,25]$. Thus, when mitochondria malfunction, they produce excessive ROS, which may be involved in carcinogenesis of cancer.

Whether gastrin could affect the production of ROS by modulating the oxidative respiratory chain of mitochondria in gastric cancer cells and thus affect cancer cell apoptosis are unclear. Herein, we aim to address these questions. Our findings can provide experimental evidence and lay the foundation for further understanding the mechanism underlying the antiapoptosis effect of gastrin in gastric cancer cells.

\section{Materials and Methods}

2.1. Cancer Cell Lines and Cell Treatment. The human gastric cancer cell line BGC823 (a gift from Laboratory of Molecular Oncology, Peking University Cancer Hospital) was used in this study. Cells were cultured with DMEM complete medium (Gibco, Grand Island, NY, USA), which contained $5 \%$ fetal bovine serum (FBS, Gibco, Grand Island, NY, USA), penicillin $(100 \mathrm{U} / \mathrm{ml})$, and streptomycin $(100 \mathrm{mg} / \mathrm{ml})$. Cells were incubated at $37^{\circ} \mathrm{C}$ with $5 \% \mathrm{CO}_{2}$. NAC (N-acetylL-cysteine) (Beyotime, Shanghai, China; $10 \mathrm{mM}$ ), the inhibitor of ROS, was added to the cells and incubated for $4 \mathrm{~h}$. Then, cells were collected for further analysis.

2.2. Construction of Plasmid and Transfection. For RNA interference, the chemically synthesized annealed siRNA duplexes were inserted between the BamHI and HindIII sites on the Psilencer ${ }^{\mathrm{TM}} 3.1-\mathrm{H} 1$ plasmid (Ambion, Austin, TX, USA) to generate gastrin-specific-interfering short hair RNA (shRNA) plasmid that was named as pshRNA plasmid, while the PsilencerTM3.1-H1 plasmid without siRNA insertion was used as empty plasmid. Cells were cultured to $60-70 \%$ confluence in $35 \mathrm{~mm}$ plates and then transfected with recombinant plasmids using the Lipofectamine2000 reagent (Invitrogen, Carlsbad, CA, USA) according to the manufacturer's instruction. Selective medium containing $400 \mathrm{~g} / \mathrm{mlG} 418$ was used to screen stable transfected cell clones after transfecting for $48 \mathrm{~h}$. The target nucleotide sequences that can interfer with the gastrin gene expression were as follows: AAGAAGAAGAAGCCTATGGAT; the sequences of shRNA designed based on the target nucleotide sequences were mentioned above: F:5'-ATCC GAAGAA GAAGCCTATGGAT TTCAAGAGA ATCCATAGGCT TCTTCTTCTT TTTTGGAAA-3'; R:5' -AGCTTTTCCAA AA AAGAAGAAGAAGCCTATGGAT TCTCTTGAA ATCCATAGGCTTCTTCTTC G-3'.
2.3. Real-Time Quantitative PCR. Total RNA was extracted from culture cells using TRIzol Reagent (Invitrogen, Carlsbad, CA, USA). Total RNA ( $5 \mu \mathrm{g})$ was reverse transcribed into cDNA with the SuperScript III First-Strand Synthesis System (Invitrogen, Carlsbad, CA, USA). The primer sequences were as follows: gastrin, 5' GAC GAG ATG CAG CGA CTA TGT 3' (sense) and 5'GGG TCT GCC ACG AGG TGT $3^{\prime}$ (antisense) and $\beta$-actin, $5^{\prime}$ CGG GAA ATC GT GCG TGA CAT T $3^{\prime}$ (sense) and $5^{\prime}$ CTA GAA GCA TTT GCG GTG GAC 3' (antisense). Real-time quantitative PCR was performed using ABI PRISM7700 Sequence Detection (Applied Biosystems, Foster City, CA, USA). $\beta$-Actin was used as an internal control. PCR reaction procedure was 28 cycles of $94^{\circ} \mathrm{C}$ denaturation for $30 \mathrm{~s}, 62^{\circ} \mathrm{C}$ annealing for $30 \mathrm{~s}$, and $72^{\circ} \mathrm{C}$ elongation for $30 \mathrm{~s}$ and a final elongation at $72^{\circ} \mathrm{C}$ for $10 \mathrm{~min}$. The relative mRNA expression levels of gastrin were calculated using the $2^{-\triangle \triangle \mathrm{Ct}}$ method.

2.4. iTRAQ and Bioinformatic Analysis. Stable cell lines with gastrin knockdown were constructed in BGC823 cells via interfering with pshRNA (BGC823-Gastrin KD), and cell lines without gastrin knockdown were constructed in BGC823 cells via interfering with empty plasmid (BGC823Ctrl cell). BGC823-Gastrin KD and BGC823-Ctrl cells were lysed in lysis buffer $(50 \mathrm{mmol} / \mathrm{L}$ Tris-Cl (pH 6.8), $100 \mathrm{mmol} / \mathrm{L}$ DTT, $2 \%$ SDS, $10 \%$ glycerol). Equal amounts of protein $(50 \mu \mathrm{g})$ were sent to Beijing Huada Protein Research \& Development Center for iTRAQ (isobaric tags for relative and absolute quantitation) analysis. The differentially expressed proteins were identified by iTRAQ. The corresponding genes that code these differentially expressed proteins were subjected to GO and KEGG analysis using the advantage $R$ software.

2.5. Mitochondrial Membrane Potential Detection. Mitochondrial membrane potential was detected by fluorescence microscopy and flow cytometry using 5,5',6,6' -tetrachloro-1,1' $, 3,3^{\prime}$-tetraethylbenzimidazolylcarbocyanine iodide (JC-1; Beyotime, Shanghai, China). BGC823-Gastrin $\mathrm{KD}$ and BGC823-Ctrl cells were cultured for $24 \mathrm{~h}$ and then incubated with $10 \mu \mathrm{M}$ JC- 1 for $2 \mathrm{~h}$ in a $\mathrm{CO}_{2}$ incubator at $37^{\circ} \mathrm{C}$. Then, cells were washed twice with PBS. Green monomers (ex485nm/em535nm) and red J-aggregates (ex560nm/em595nm, a sensitive marker of $\Delta \psi \mathrm{m})$ were detected by laser confocal scanning microscope (TCS- SP5; Leica, Mannheim, Germany) and by flow cytometry, respectively (BD FACSAria, San Jose, CA, USA).

2.6. Dichloro-Dihydro-Fluorescein Diacetate (DCFH-DA) Assay. ROS level in gastric cancer cells was detected by the ROS detection kit (Beyotime Biotechnology, Shanghai, China). The DCFH-DA probe was diluted with a serumfree medium at 1:1000, and the work concentration was $10 \mu \mathrm{M}$. After removal of culture medium, $1 \mathrm{ml}$ of diluted DCFH-DA probe was added to cells and incubated at $37^{\circ} \mathrm{C}$ for $20 \mathrm{~min}$. Later, cells were washed three times with serum-free medium to completely remove DCFH-DA probe that did not enter the cells and then observed by laser confocal scanning microscope (TCS- SP5; Leica, Mannheim, 


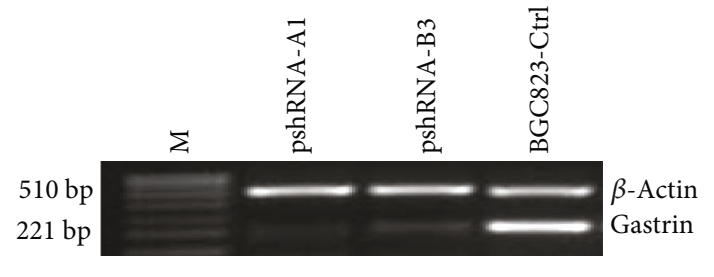

(a)

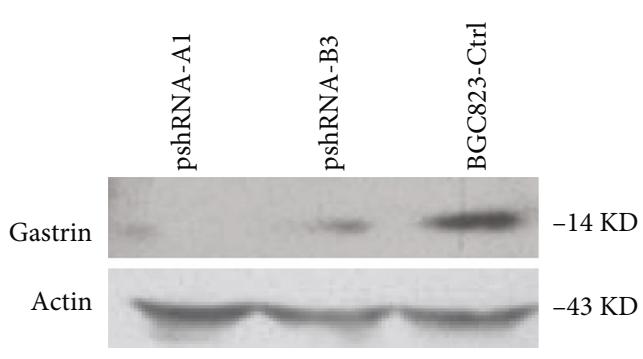

(c)

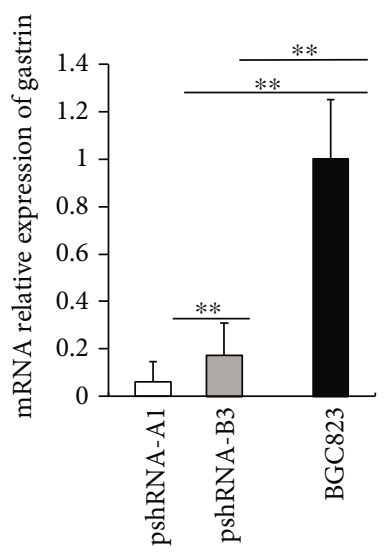

(b)

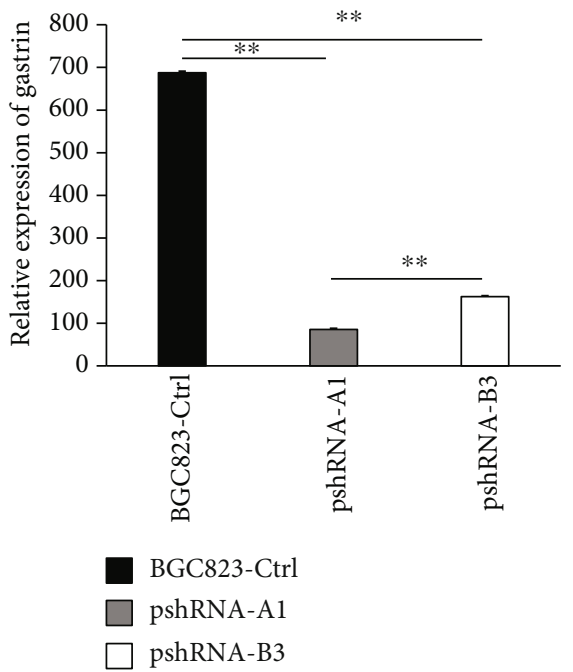

(d)

FIGURE 1: Knockdown of the gastrin expression in BGC823 cell line by RNA interference. (a) RT-PCR analysis detected more obvious interfering effect on gastrin in cell clones pshRNA-A1 and pshRNA-B3. (b) Level of gastrin mRNA was measured by real-time RT-PCR. The error bars represent the standard deviations of triplicate experiments, ${ }^{* *} P<0.001$. (c) Level of gastrin protein in clones pshRNA-A1, pshRNA-B3, and BGC823-Ctrl by Western blotting. (d) Grayscale scanning results of Western blot, ${ }^{* *} P<0.001$.

Germany) and analyzed by flow cytometry (BD FACSAria, San Jose, CA, USA).

2.7. Flow Cytometry for Analysis of Apoptosis. To detect the apoptosis of cells, Annexin V-FITC apoptosis detection kit (Beijing Baosai Biotech, Beijing, China) was used. Briefly, cells were collected and suspended in prechilled PBS. After three times washing with PBS, cells were resuspended in $200 \mu \mathrm{l}$ binding buffer with $10 \mu \mathrm{l}$ Annexin V-FITC and incubated in dark for $15 \mathrm{~min}$ at room temperature. Later, $300 \mu \mathrm{l}$ binding buffer with $5 \mu \mathrm{l}$ PI solution was added into the cell suspension. Then, the apoptotic rate of gastric cancer cells was detected by flow cytometer (BD FACSAria, San Jose, CA, USA) within an hour.

2.8. Western Blot Analysis. Two stable clones of BGC823Gastrin $\mathrm{KD}$ cells were selected and, respectively, named as PshrNa-A1 and PshrNa-B3. BGC823-Ctrl cells and untreated BGC823 cells were taken as control. These cells were sub- jected to lysis ( $50 \mathrm{mmol} / \mathrm{L}$ Tris-Cl (pH 6.8), $100 \mathrm{mmol} / \mathrm{L}$ DTT, 2\% SDS, and 10\% glycerol). Equal amounts of protein $(50 \mu \mathrm{g})$ were then separated on $12 \%$ SDS-PAGE and transferred to PVDF membrane (Pharmacia, (GE), USA). The membrane was blocked with $5 \%$ nonfat milk for $1 \mathrm{~h}$ and then incubated with the specific antibody: anti-gastrin (Abcam, Cambridge, MA, USA) (1:500), anti-I $\kappa \mathrm{B}-\alpha$ (Santa Cruz, CA, USA) (1:500), anti-p-I $\kappa$ B- $\alpha$ (Santa Cruz, CA, USA) (1:500), anti-NF- $\kappa$ B (Santa Cruz, CA, USA) (1:500), anti-Bcl-2 (US Biological, Boston, USA) (1:500), anti-Bax (Epitomics, Burlingame, CA, USA) (1:500), anti-COX17(Abcam, Cambridge, MA, USA) (1:500), anti-COX5B(Abcam, Cambridge, MA, USA) (1:500), anti-ATP5J(Abcam, Cambridge, MA, USA) $(1: 500)$, and anti- $\beta$-actin (Santa Cruz, CA, USA) $(1: 20000)$ overnight at $4^{\circ} \mathrm{C}$. Horseradish peroxidase-conjugated secondary antibodies (Santa Cruz, CA, USA) were used at a 1:2000 concentration. Color development was performed with the ECL system (GE Health Care, Little Chalfont, Buckinghamshire, UK). 


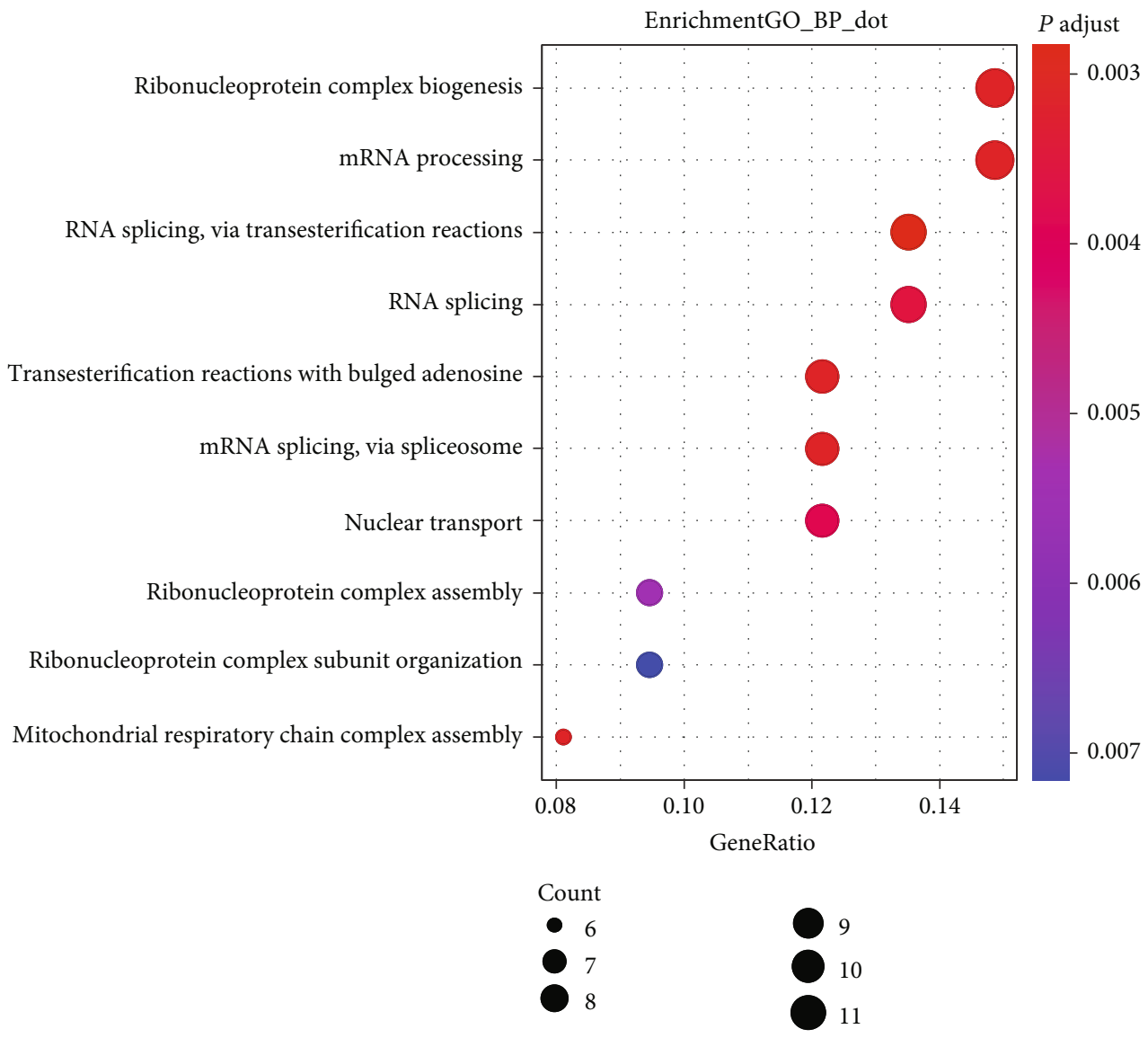

(a)

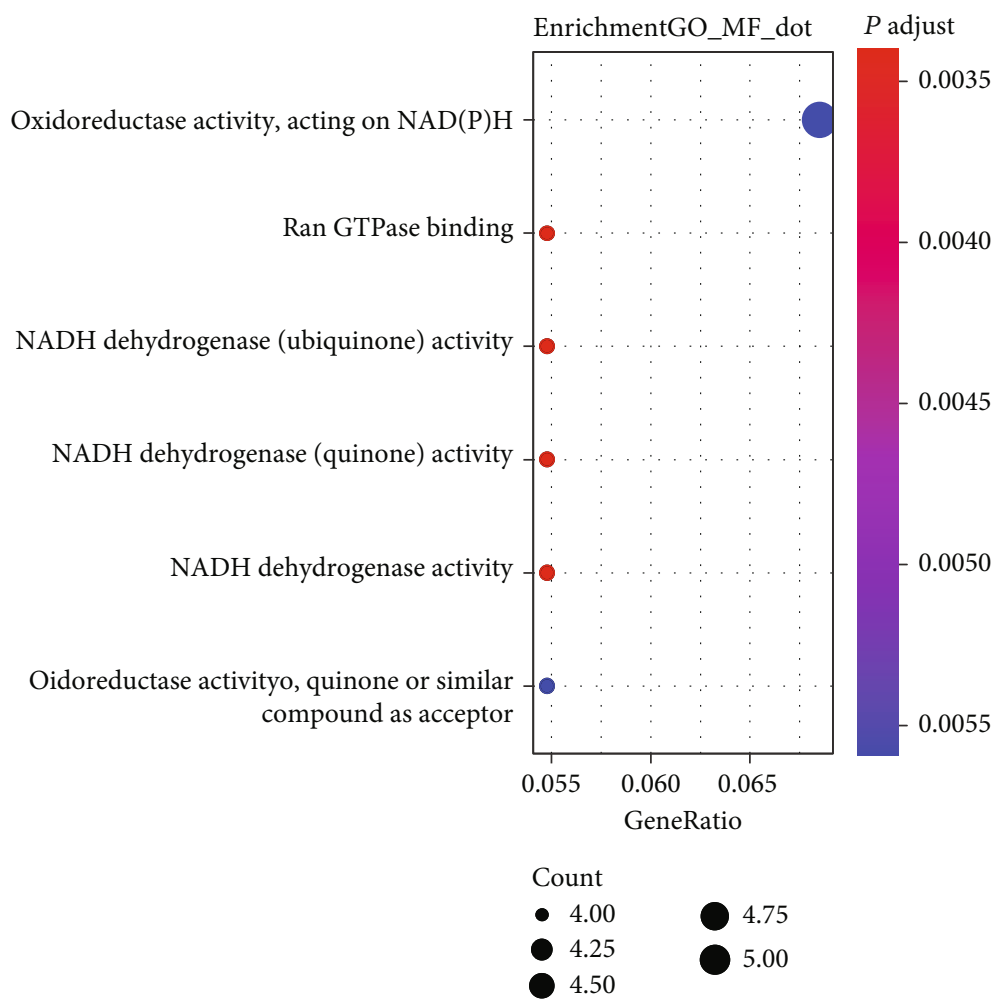

(b)

Figure 2: Continued. 


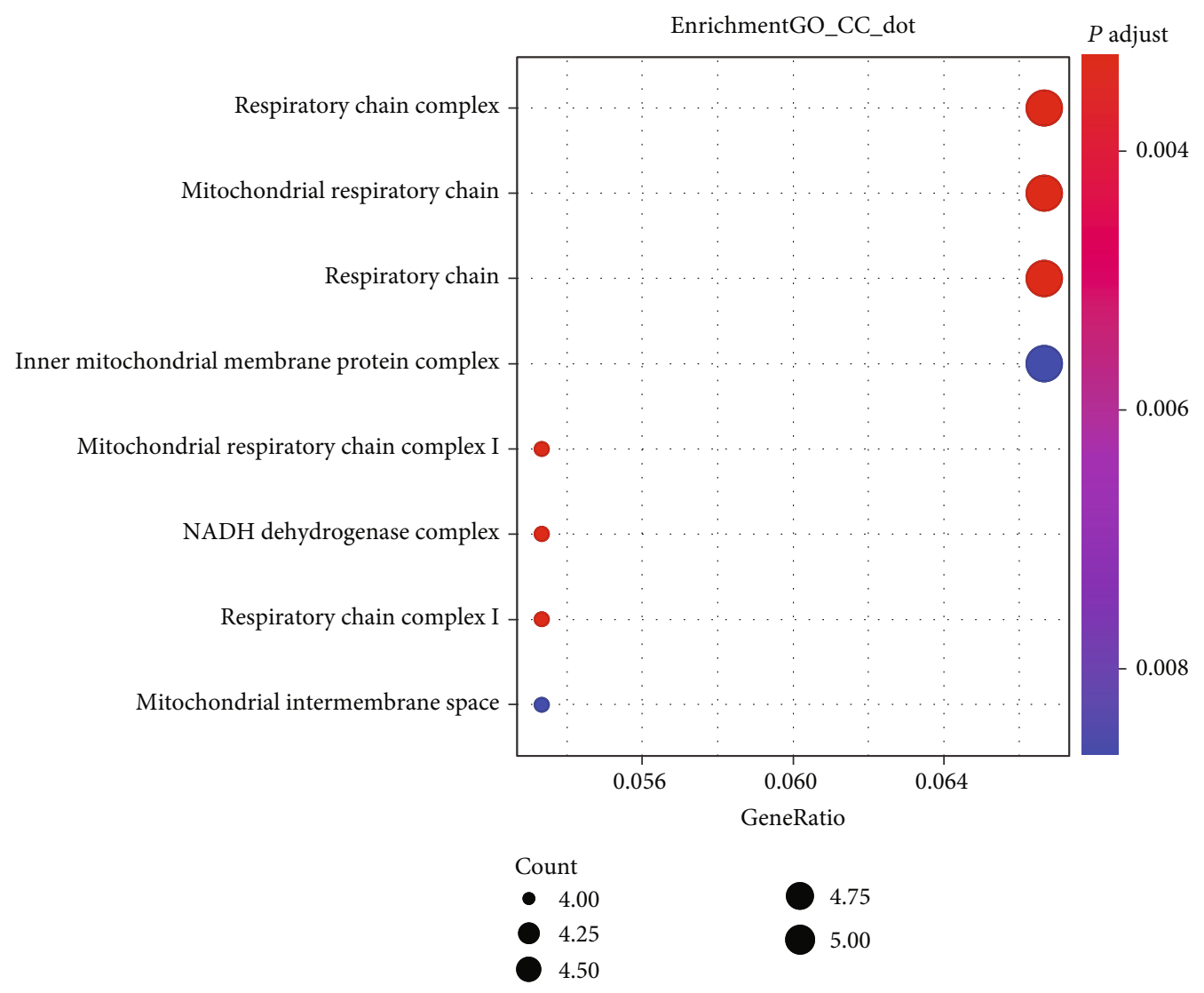

(c)



(d)

Figure 2: Continued. 



(e)

FIGURE 2: Knockdown of gastrin results in mitochondrial dysfunction in gastric cancer cells. Enrichment analysis of the differentially expressed genes identified by iTRAQ in gastrin knockdown gastric cancer cells. The bubble diagrams display the enrichment results of the 107 genes differentially expressed in gastrin knockdown gastric cancer cells compared with control cells. (a) Biological processes. (b) Molecular functions. (c) Cellular components. (d) KEGG pathway analysis. (e) Western blot was used to detect the expression of mitochondrial-related proteins (COX17, COX5B, and ATP5J) in BGC823 cells after interfering with gastrin, ${ }^{* *} P<0.001$ vs BGC823-Ctrl.

2.9. Statistical Analysis. Data was analyzed by using statistical software SPSS19.0 (IBM, Armonk, NY, USA). The chi-square test was used for counting data comparison. Differences between two groups were evaluated using two-sided unpaired Student's $t$-test. Comparison of more than two groups was performed with one-way ANOVA followed by Tukey's multiple comparison test. Statistical significance was set at the level of $P<0.05$.

\section{Results}

3.1. The pshRNA Effectively and Persistently Suppresses the Gastrin Expression in BGC823 Cells. We successfully constructed pshRNA recombinant plasmids targeting gastrin. After 22 days of selection, positive cell clone was obtained. To examine whether shRNA induces gene silencing, qRTPCR and Western blot were performed to detect the gastrin expression. We found that the gastrin mRNA expression was significantly downregulated in two clones of pshRNAA1 and pshRNA-B3 (Figures 1(a) and 1(b)). Western blot analysis showed the dramatic decrease of gastrin protein levels in two clones compared to control cells BGC823-Ctrl (Figures 1(c) and 1(d)). Because the downregulation of the mRNA and protein expression level of gastrin was more significantly in pshRNA-A1 than that in pshRNA-B3, we selected the clone with stable pshRNA-A1 transfection to represent BGC823-gastrin KD for follow-up experiments.

\subsection{Knockdown of Gastrin Results in Expression Level Changes} of Proteins Involved in the Oxidative Phosphorylation Function of Cell Mitochondria in Gastric Cancer Cells. iTRAQ analysis is one of the mass spectrometry-driven protein quantification methods and always applied to identify differentially expressed proteins between groups in a high throughput manner. Here, iTRAQ analysis was used to identify the differentially expressed proteins between BGC823-Gastrin KD and BGC823-Ctrl cells. We found 107 significantly differently expressed proteins (either overexpression or downexpression) when gastrin was knocked out. GO analysis showed that these differentially expressed proteins mainly located in respiratory chain complex (Figures 2(a)-2(c)), where they participate primarily in mitochondrial respiratory chain complex assembly. They also had the oxidoreductase activity. KEGG pathway analysis showed that these proteins were enriched in oxidative phosphorylation pathways (Figure 2(d)).

Western blots were performed for the proteins of COX17, COX5B, and ATP5J, which are important components of mitochondrial oxidation respiratory chain, to confirm iTRAQ results. As shown in Figure 2(e), COX17, COX5B, and ATP5J were overexpressed after gastrin knockdown in gastric cancer $(P<0.01)$. These results indicate for the first time that the knockdown of gastrin in gastric cancer cells may affect the oxidative phosphorylation function of cell mitochondria via upregulating the COX17, COX5B, and ATP5J expression level.

\subsection{Knockdown of Gastrin Affects Mitochondrial Membrane} Potential. Since the mitochondrial associated protein was overexpressed in gastrin-silenced cells, we hypothesize that there may be some mitochondrial defects in cancer cells. So, we used JC-1 staining to detect mitochondrial membrane potential. As shown in Figure 3(a), in BGC823-Ctrl cells, the green fluorescence was stronger, and the red fluorescence was weaker. However, after interfering with the gastrin expression, the red light fluorescence enhanced, and the green 




(a)


(b)

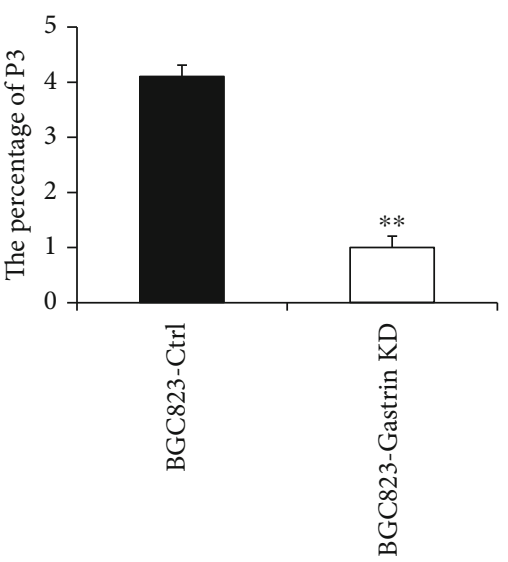

BGC823-Ctrl

BGC823-Gastrin KD

(c)

FIGURE 3: Knockdown of gastrin affects the mitochondrial membrane potential. (a) JC-1 staining was detected cell mitochondrial membrane potential and analyzed by laser-scanning confocal microscopy. The images showed representative fluorescence micrographs of JC-1-stained gastrin KD cells and control cells. (b) Mitochondrial membrane potential evaluated by JC-1 staining and analyzed by flow cytometry. Cell populations with higher and lower JC-1 aggregated staining are marked with high percentage of p3 and low percentage of p3, respectively. A decrease in the $\mathrm{p} 3$ indicates a rise in mitochondrial membrane potential. Each column represents the mean \pm SD of three independent experiments $\left({ }^{* *} P<0.01\right.$ vs BGC823-Ctrl).

fluorescence was weakened in BGC823-Gasrin KD cells. Consistently, flow cytometry showed increased red fluorescence intensity, decreased green fluorescence intensity, and increased red/green fluorescence intensity ratio in BGC823Gasrin KD cells (Figures 3(b) and 3(c), $P<0.01$ ). These results indicate that gastrin knockdown increases mitochondrial membrane potential in gastric cancer cells.

\subsection{Knockdown of Gastrin Promotes Apoptosis of Gastric} Cancer Cells. Mitochondria are important organelles for regulating cell apoptosis. To evaluate the effect of gastrin knockdown on apoptosis of gastric cancer cells, the Annexin V-FITC apoptosis assay was done. Results showed that the apoptotic rate of gastric cancer cells was significantly higher in BGC823-Gasrin KD cells than that in
BGC823-Ctrl cells, which were $4.2 \%$ and $1.8 \%$, respectively (Figures 4(a) and 4(b), $P<0.05)$. This data indicates that knockdown of gastrin significantly promoted apoptosis of gastric cancer cells.

\subsection{Knockdown of Gastrin Reduces the Level of Mitochondrial} ROS in Gastric Cancer Cells. As known, intracellular ROS is mainly generated in mitochondria, and thus we speculate that gastrin knockdown may affect the ROS generation. To verify this speculation, the ROS level in mitochondria of gastric cancer cells was detected by DCFH-DA probe. The green fluorescence intensity is proportional to the level of intracellular ROS. Both of confocal microscope (Figure 5(a)) and flow cytometry (Figures 5(b) and 5(c)) results showed that green fluorescence intensity was significantly decreased after 




(a)

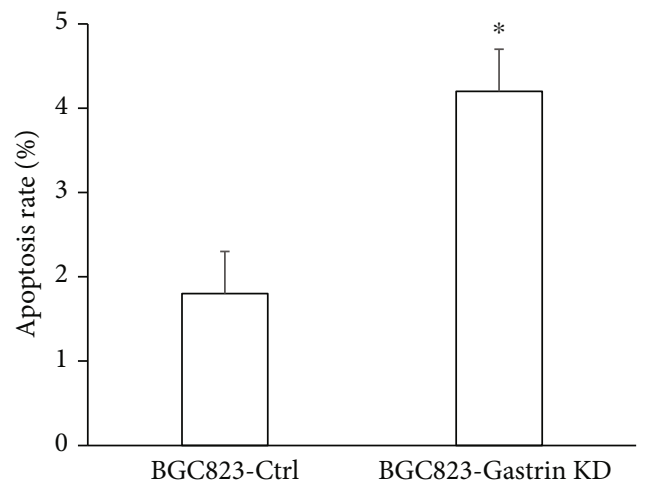

(b)

Figure 4: Knockdown of gastrin promotes apoptosis of gastric cancer cells. (a) The apoptosis was detected by flow cytometry. (b) The apoptotic rate of gastric cancer was comparatively analyzed $\left({ }^{*} P<0.05\right.$ vs BGC823-Ctrl).

knockdown of gastrin in BGC823 $(P<0.01)$, indicating that intracellular ROS production decreased after knockdown of the gastrin expression.

3.6. Knockdown of Gastrin Affects the Expression of Mitochondrial Apoptotic-Related Proteins in Gastric Cancer Cells. Western blot was performed to determine the effect of gastrin knockdown on protein expressions of $\mathrm{I} \kappa \mathrm{B}-\alpha, \mathrm{p}-$ $\mathrm{I} \kappa \mathrm{B}-\alpha, \mathrm{NF}-\kappa \mathrm{B}-\mathrm{p} 65, \mathrm{Bax}$, and $\mathrm{Bcl}-2$. The results showed that the expression of $\mathrm{I} \kappa \mathrm{B}-\alpha$ was significantly upregulated, while p-I $\kappa$ B- $\alpha$ and NF- $\kappa$ B-p65 were significantly downregulated after knockdown of gastrin in gastric cancer cells (Figure 6(a)) $(P<0.05)$. These data suggest that gastrin can promote THE NF- $\kappa \mathrm{B}$ activity.

Since both expression levels of $\mathrm{Bax}$ and $\mathrm{Bcl}-2$ proteins are regulated by NF- $\kappa \mathrm{B}$ [26], we used Western blot analysis to test whether gastrin-induced NF- $\kappa \mathrm{B}$ activation affects the $\mathrm{Bax}$ and $\mathrm{Bcl}-2$ expression. Our results showed that gastrin knockdown caused reduction of Bcl-2 levels and elevation of Bax levels (Figure 6(a)).

To further confirm whether the above protein expression change induced by gastrin is associated with ROS generation, we pretreated cells with the antioxidant NAC, which is a ROS scavenger. Compared with untreated BGC823-Ctrl cells, the
Bax expression level was upregulated, and the Bcl-2 expression level was downregulated in BGC823-Ctrl cells pretreated with NAC. Moreover, Bax and Bcl-2, respectively, had similar expression levels between BGC823-Gasrin KD cells and GC823-Ctrl cells treated with NAC (Figure 6(b), $P<0.01$ ), indicating that gastrin-induced $\mathrm{Bax}$ and $\mathrm{Bcl}-2$ expression changes are ROS-dependent.

\section{Discussion}

In this study, we found that knockdown of gastrin in gastric cancer cells could result in the overexpression of COX17, COX5B, and ATP5J proteins, elevation of mitochondrial membrane potential, and reduction of ROS production in gastric cancer cells. Moreover, our results also showed that gastrin knockdown caused reduction of Bcl-2 levels and elevation of Bax levels in a ROS-dependent manner, indicating that gastrin knockdown promotes gastric cancer cell apoptosis by modulating the expression levels of Bax and Bcl-2.

Gastrin is a traditional oncogenic factor. It could promote angiogenesis by activating HIF- $1 \alpha / \beta$-catenin/VEGF signaling in gastric cancer [27]. In addition, it is proved that gastrin functions as a stimulator of the metastasis of gastric carcinoma through the $\beta$-catenin-TCF4 pathway [28]. 

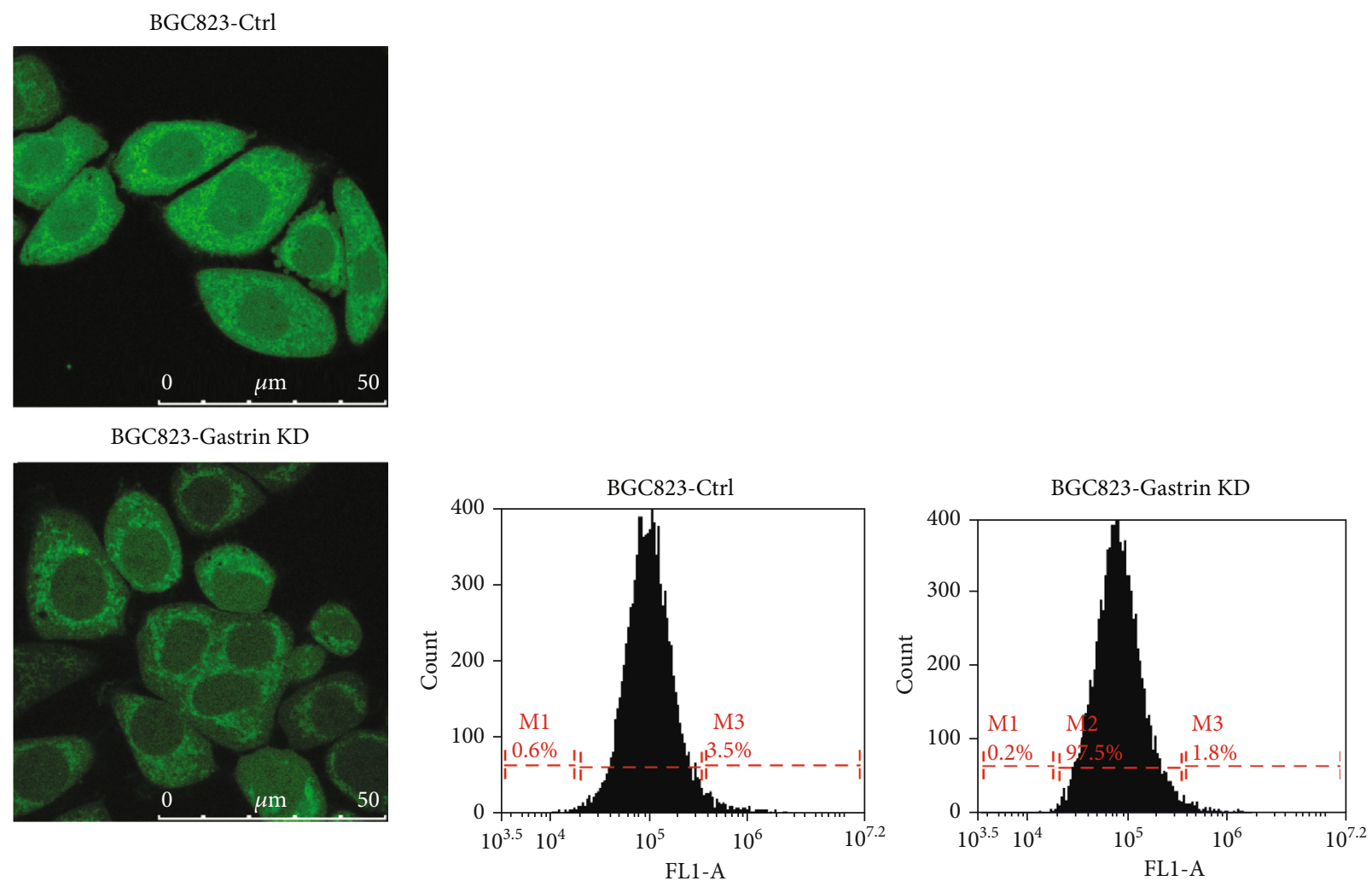

(a)

(b)

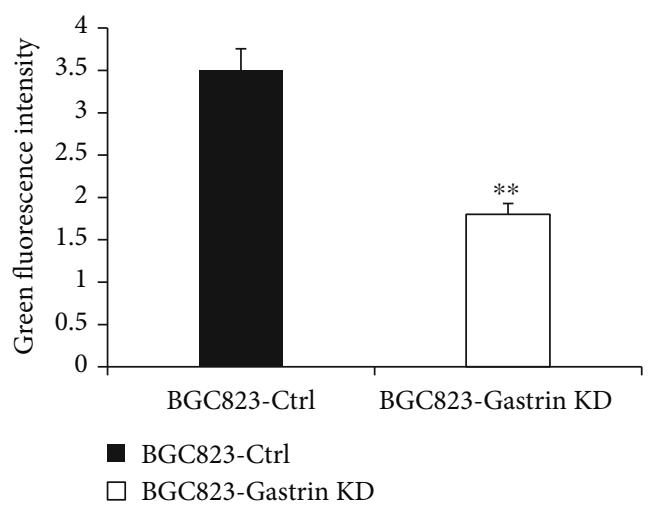

(c)

FIGURE 5: Knockdown of gastrin inhibits ROS production in gastric cancer cells. (a) Representative photos of the DCFH-DA probe assay under laser-scanning confocal microscopy (scale bar $50 \mu \mathrm{M}$ ). (b) Flow cytometry was performed to detect the ROS level using DCFH-DA molecular probe. (c) Statistical results of the ROS level. Data are expressed as means \pm SD $\left({ }^{* *} P<0.01\right.$ vs BGC823-Ctrl).

Moreover, gastrin could regulate autophagy of gastric cancer cells through the AMPK $\alpha /$ Ras/Raf/MEK/ERK pathway and plays a procancer role [29]. Although its role as an oncogenic factor has been very clear, there has been no new breakthrough in recent years. To further characterize the function of gastrin in GC development, we investigated protein expression changes by performing iTRAQ analysis. We unexpectedly detected that knockdown of gastrin resulted in the overexpression of several mitochondrial respiratory chain-related proteins and elevated mitochondrial membrane potential in gastric cancer cells.

Recently, it is reported that there is a close relationship between mitochondria and tumor development $[26,30]$. Mito- chondrial dysfunction, especially the abnormal expression of mitochondrial respiratory chain-related proteins, is reported to be closely related to oncogenesis, development, invasion, and metastasis of various tumors [31, 32]. For example, it is reported that the expression level of NDUFA13, a subunit of mitochondrial respiratory chain complex $\mathrm{I}$, decreases in a variety of tumors [33], such as lung [34], gastric [35], and liver [36] tumors, which promotes tumorigenesis by inhibiting tumor cell apoptosis [37]. Here, we found the effect of gastrin on the expression levels of COX17, COX5B, and ATP5J proteins that are involved in the mitochondrial respiratory chain, which may reveal one of the important mechanisms of gastrin as a cancer promoting factor. 



BGC823-Ctrl

$\square$ pshRNA-Gastrin KD

(a)
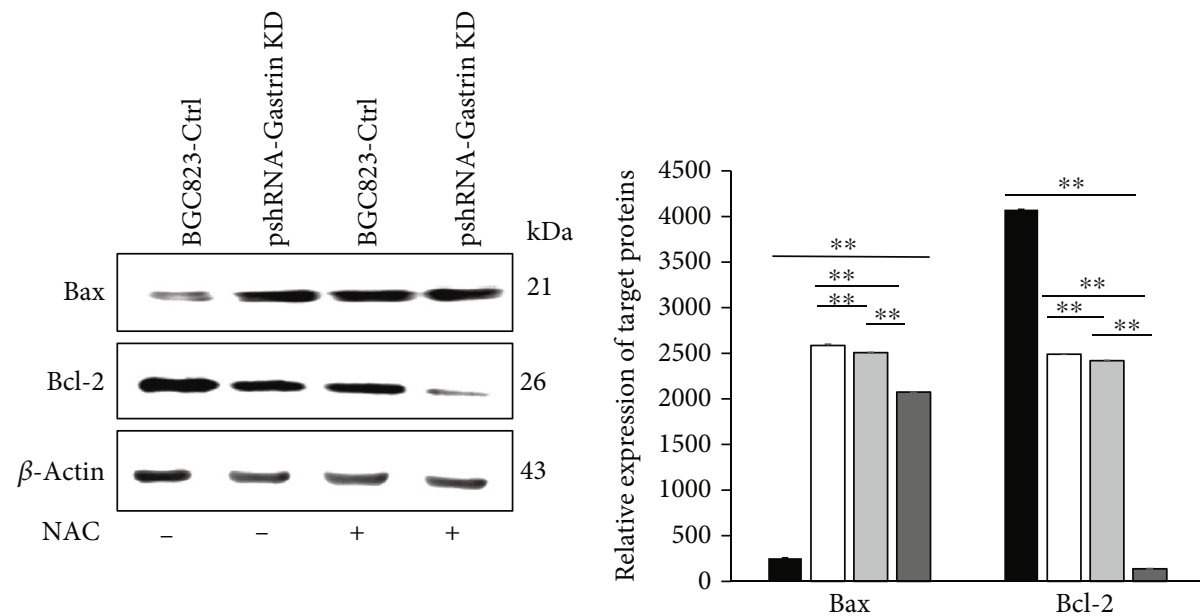

BGC823-Ctrl-NAC_negative

BGC823-Ctrl-NAC_positive

pshRNA-Gastrin KD-NAC_negative

pshRNA-Gastrin KD-NAC_positive

(b)

FIGURE 6: Knockdown of gastrin inhibits the expression of mitochondrial apoptotic-related protein in gastric cancer cells. (a) Western blot analysis of NF- $\kappa \mathrm{B}$ and $\mathrm{Bcl}-2$ family proteins in gastrin $\mathrm{KD}$ cells compared with control cells. $\beta$-Actin was used as a loading control. Data are expressed as means $\pm \mathrm{SD}\left({ }^{* *} P<0.01\right)$. (b) Western blot analysis of Bcl-2 family proteins in gastrin KD cells compared with control cells after pretreatment with NAC. Data are expressed as means $\pm \operatorname{SD}\left({ }^{* *} P<0.01\right)$.

ROS is the product of normal oxidative metabolism of cells, and endogenous ROS are mainly produced by mitochondria [38]. Dysfunction of the mitochondrial electron transport chain (such as blocking the function of complexIor complex II) significantly promoted ROS generation [39], resulting in accumulation of ROS in mitochondria and cytoplasm, leading to oxidative damage and abnormal cell function. Under hypoxia condition, the expression of NDUFA5,
NDUFS6, NDUFA9 (mitochondrial respiratory chain complex I), cytochrome B (complex III), mitochondrialencoded subunit I (COXI), and nuclear-encoded subunit IV (COXIV) (complex IV) decreased in TE-1 cells (Esophageal squamous cell carcinoma cell line), which promoted ROS generation and further accelerated the proliferation of tumor cells [40]. We, thus, propose that gastrin is the initi$\mathrm{al} /$ critical event that results in mitochondria ROS elevation 
and affects cell apoptosis. As expected, our data demonstrate that BGC823-Gastrin KD cell, which had mitochondrial dysfunction, resulted in reduction of ROS production via upregulating COX17, COX5B, and ATP5J expression and increasing mitochondria potential, suggesting that gastrin is one of key factors that influence the ROS level.

Compelling evidence suggests that the increased ROS stress in cancer cells has a pivotal role in the acquisition of the hallmarks of cancer, including disruption of cell death signaling [41], since NF- $\kappa \mathrm{B}$ is a redox-sensitive transcription factor that is activated by increased levels of ROS [42]. And $\mathrm{NF}-\kappa \mathrm{B}$ also has been shown to induce the expression of some members of the antiapoptotic proteins [43]. Thus, we examined whether knockdown of gastrin is associated with deactivation of $\mathrm{NF}-\kappa \mathrm{B}$ in a ROS-dependent manner in gastric cancer cells. Our data showed that gastrin knockdown upregulated the protein expression level of $\mathrm{I} \kappa \mathrm{B}-\mathrm{a}$ and downregulated NF- $\kappa \mathrm{B}$ activation in GC cells depending on low levels of ROS. Thus, NF- $\kappa \mathrm{B}$ pathways may be involved in the gastrin mediated regulation of apoptosis. We also observed a decrease in the expression of antiapoptotic $\mathrm{Bcl}-2$ proteins and an increase in proapoptotic Bax in gastrin knockdown cells. Furthermore, compared with untreated BGC823-Ctrl cells, the Bax expression level was upregulated, and Bcl-2 expression level was downregulated in BGC823-Ctrl cells pretreated with NAC. Moreover, Bax and Bcl-2 had similar expression level changes between BGC823-Gasrin KD cells and GC823-Ctrl cells treated with NAC, indicating that gastrin-induced Bax and Bcl-2 expression changes are ROSdependent, and gastrin knockdown may promote apoptosis via upregulating Bax and downregulating Bcl-2.

\section{Conclusions}

To sum up, gastrin knockdown upregulates the expression of mitochondrial respiratory chain proteins, including COX17, COX5B, and ATP5J; increases the mitochondrial potential; and decreases the production of ROS. Moreover, knockdown of gastrin is associated with deactivation of NF- $\kappa \mathrm{B}$ in a ROSdependent manner in gastric cancer cells and upregulation of Bax and downregulation of $\mathrm{Bcl}-2$, thus promoting apoptosis.

\section{Data Availability}

The data are available from the corresponding author.

\section{Conflicts of Interest}

The authors declare that there is no conflict of interest regarding the publication of this paper.

\section{Acknowledgments}

This study was supported by the National Natural Science Foundation of China (No. 81960451), Qinghai Science and Technology Department Natural Foundation (No. 2019-ZJ7055), and Qinghai University Medical College Team Project Foundation (No. 2018-kyt-2).

\section{References}

[1] L. Friis-Hansen, F. Sundler, Y. Li et al., "Impaired gastric acid secretion in gastrin-deficient mice," American Journal of Physiology-Gastrointestinal and Liver Physiology, vol. 274, no. 3, pp. G561-G568, 1998.

[2] S. A. Watson, A. M. Grabowska, M. el-Zaatari, and A. Takhar, "Gastrin - active participant or bystander in gastric carcinogenesis?," Nature Reviews Cancer, vol. 6, no. 12, pp. 936-946, 2006.

[3] J. P. Smith, L. K. Fonkoua, and T. W. Moody, "The role of gastrin and CCK receptors in pancreatic cancer and other malignancies," International Journal of Biological Sciences, vol. 12, no. 3, pp. 283-291, 2016.

[4] Q. Zhang, Z. Lv, L. P. Sun, N. Dong, C. Xing, and Y. Yuan, "Clinical significance of serum markers reflecting gastric function andH. pyloriinfection in colorectal cancer," Journal of Cancer, vol. 10, no. 10, pp. 2229-2236, 2019.

[5] T. W. Moody, B. Nuche-Berenguer, P. Moreno, and R. T. Jensen, "CI-988 inhibits EGFR transactivation and proliferation caused by addition of CCK/gastrin to lung cancer cells," Journal of Molecular Neuroscience, vol. 56, no. 3, pp. 663-672, 2015.

[6] Y. Zhang, C. Zhu, and X. Lu, "Advances in serum biomarkers for early diagnosis of gastric cancer," Zhejiang Da Xue Xue Bao Yi Xue Ban, vol. 48, no. 3, pp. 326-333, 2019.

[7] S. V. Rao, G. Solum, B. Niederdorfer, K. G. Nørsett, G. Bjørkøy, and L. Thommesen, "Gastrin activates autophagy and increases migration and survival of gastric adenocarcinoma cells," BMC Cancer, vol. 17, no. 1, p. 68, 2017.

[8] N. Osborne, R. Sundseth, J. Burks et al., "Gastrin vaccine improves response to immune checkpoint antibody in murine pancreatic cancer by altering the tumor microenvironment," Cancer Immunology, Immunotherapy, vol. 68, no. 10, pp. 1635-1648, 2019.

[9] L. Feng, J. Zhu, W. Sun, J. Zhao, and Y. Liu, "Expressions of gastrin and apoptosis-associated proteins involved in mitochondrial pathway in gastric cancer tissues and the clinical significance," Xi Bao Yu Fen Zi Mian Yi Xue Za Zhi, vol. 33, no. 11, pp. 1557-1561, 2017.

[10] Y. Shiomi, M. Yoshimura, K. Kuki, Y. Hori, and T. Tanaka, "Z360 Suppresses Tumor Growth in MIA PaCa-2-bearing Mice via Inhibition of Gastrin-induced Anti-Apoptotic Effects," Anticancer Research, vol. 37, no. 8, pp. 4127-4137, 2017.

[11] H. L. Waldum, L. Sagatun, and P. Mjønes, "Gastrin and gastric cancer," Frontiers in Endocrinology, vol. 8, p. 1, 2017.

[12] H. L. Waldum and J. F. Rehfeld, "Gastric cancer and gastrin: on the interaction of helicobacter pylori gastritis and acid inhibitory induced hypergastrinemia," Scandinavian Journal of Gastroenterology, vol. 54, no. 9, pp. 1118-1123, 2019.

[13] S. Maglioni and N. Ventura, "C. elegans as a model organism for human mitochondrial associated disorders," Mitochondrion, vol. 30, pp. 117-125, 2016.

[14] C. Yee, W. Yang, and S. Hekimi, "The intrinsic apoptosis pathway mediates the pro-longevity response to mitochondrial ROS in C. elegans," Cell, vol. 157, no. 4, pp. 897-909, 2014.

[15] S. Hekimi, Y. Wang, and A. Noë, "Mitochondrial ROS and the effectors of the intrinsic apoptotic pathway in aging cells: the discerning killers!," Frontiers in Genetics, vol. 7, p. 161, 2016.

[16] N. Demaurex, D. Poburko, and M. Frieden, "Regulation of plasma membrane calcium fluxes by mitochondria," 
Biochimica et Biophysica Acta, vol. 1787, no. 11, pp. 13831394, 2009.

[17] K. Oxenoid, Y. Dong, C. Cao et al., "Architecture of the mitochondrial calcium uniporter," Nature, vol. 533, no. 7602, pp. 269-273, 2016.

[18] S. Gururaja Rao, "Mitochondrial changes in cancer," Handbook of Experimental Pharmacology, vol. 240, pp. 211-227, 2016.

[19] D. Hanahan and R. A. Weinberg, "Hallmarks of cancer: the next generation," Cell, vol. 144, no. 5, pp. 646-674, 2011.

[20] W. H. Koppenol, P. L. Bounds, and C. V. Dang, "Otto Warburg's contributions to current concepts of cancer metabolism," Nature Reviews Cancer, vol. 11, no. 5, pp. 325-337, 2011.

[21] A. M. Otto, "Warburg effect(s)-a biographical sketch of Otto Warburg and his impacts on tumor metabolism," Cancer \& Metabolism, vol. 4, no. 1, p. 5, 2016.

[22] S. Y. Han, Y. J. Jeong, Y. Choi, S. K. Hwang, Y. S. Bae, and Y. C. Chang, "Mitochondrial dysfunction induces the invasive phenotype, and cell migration and invasion, through the induction of AKT and AMPK pathways in lung cancer cells," International Journal of Molecular Medicine, vol. 42, no. 3, pp. 1644-1652, 2018.

[23] H. J. Kim, P. Maiti, and A. Barrientos, "Mitochondrial ribosomes in cancer," Seminars in Cancer Biology, vol. 47, pp. 67-81, 2017.

[24] J. Lu, M. Tan, and Q. Cai, “The Warburg effect in tumor progression: mitochondrial oxidative metabolism as an antimetastasis mechanism," Cancer Letters, vol. 356, no. 2, pp. 156-164, 2015.

[25] E. Gottlieb and I. P. Tomlinson, "Mitochondrial tumour suppressors: a genetic and biochemical update," Nature Reviews Cancer, vol. 5, no. 11, pp. 857-866, 2005.

[26] L. Li, W. Wu, W. Huang, G. Hu, W. Yuan, and W. Li, "NF- $\kappa$ B RNAi decreases the Bax/Bcl-2 ratio and inhibits TNF- $\alpha$ induced apoptosis in human alveolar epithelial cells," Inflammation Research, vol. 62, no. 4, pp. 387-397, 2013.

[27] S. Toyokuni, K. Okamoto, J. Yodoi, and H. Hiai, "Persistent oxidative stress in cancer," FEBS Letters, vol. 358, no. 1, pp. 1-3, 1995.

[28] J. T. Hancock, R. Desikan, and S. J. Neill, "Role of reactive oxygen species in cell signalling pathways," Biochemical Society Transactions, vol. 29, no. 2, pp. 345-349, 2001.

[29] B. Kumar, S. Koul, L. Khandrika, R. B. Meacham, and H. K. Koul, "Oxidative stress is inherent in prostate cancer cells and is required for aggressive phenotype," Cancer Research, vol. 68, no. 6, pp. 1777-1785, 2008.

[30] E. Tang, Y. Wang, T. Liu, and B. Yan, "Gastrin promotes angiogenesis by activating HIF- $1 \alpha / \beta$-catenin/VEGF signaling in gastric cancer," Gene, vol. 704, pp. 42-48, 2019.

[31] K. Zhuang, Y. Yan, X. Zhang, J. Zhang, L. Zhang, and K. Han, "Gastrin promotes the metastasis of gastric carcinoma through the $\beta$-catenin/TCF-4 pathway," Oncology Reports, vol. 36, no. 3, pp. 1369-1376, 2016.

[32] Z. Kun, G. Hanqing, T. Hailing et al., "Gastrin enhances autophagy and promotes gastric carcinoma proliferation via inducing AMPK $\alpha$," Oncology Research, vol. 25, no. 8, pp. 1399-1407, 2017.

[33] L. Iommarini, A. Ghelli, G. Gasparre, and A. M. Porcelli, "Mitochondrial metabolism and energy sensing in tumor progression," Biochimica et Biophysica Acta - Bioenergetics, vol. 1858 , no. 8 , pp. 582-590, 2017.
[34] L. Putignani, S. Raffa, R. Pescosolido et al., "Preliminary evidences on mitochondrial injury and impaired oxidative metabolism in breast cancer," Mitochondrion, vol. 12, no. 3, pp. 363-369, 2012.

[35] The PLOS ONE Editors, "Retraction: mitochondrial dysfunction promotes breast cancer cell migration and invasion through HIF1 $\alpha$ accumulation via increased production of reactive oxygen species," PLoS One, vol. 13, no. 10, article e0205288, 2018.

[36] E. Gaude and C. Frezza, "Defects in mitochondrial metabolism and cancer," Cancer \& Metabolism, vol. 2, no. 1, p. 10, 2014.

[37] P. L. Chen, C. F. Chen, Y. Chen et al., "Mitochondrial genome instability resulting from SUV3 haploinsufficiency leads to tumorigenesis and shortened lifespan," Oncogene, vol. 32, no. 9, pp. 1193-1201, 2013.

[38] S. C. Nallar and D. V. Kalvakolanu, "GRIM-19: a master regulator of cytokine induced tumor suppression, metastasis and energy metabolism," Cytokine \& Growth Factor Reviews, vol. 33, pp. 1-18, 2017.

[39] X. Y. Fan, Z. F. Jiang, L. Cai, and R. Y. Liu, "Expression and clinical significance of GRIM-19 in lung cancer," Medical Oncology, vol. 29, no. 5, pp. 3183-3189, 2012.

[40] X. Bu, C. Zhao, W. Wang, and N. Zhang, "GRIM-19 inhibits the STAT3 signaling pathway and sensitizes gastric cancer cells to radiation," Gene, vol. 512, no. 2, pp. 198-205, 2013.

[41] D. Kong, L. Zhao, Y. du et al., "Overexpression of GRIM-19, a mitochondrial respiratory chain complex I protein, suppresses hepatocellular carcinoma growth," International Journal of Clinical and Experimental Pathology, vol. 7, no. 11, pp. 74977507, 2014.

[42] S. Kalakonda, S. C. Nallar, S. Jaber et al., "Monoallelic loss of tumor suppressor GRIM-19 promotes tumorigenesis in mice," Proceedings of the National Academy of Sciences of the United States of America, vol. 110, no. 45, pp. E4213-E4222, 2013.

[43] H. Wei and X. Cong, "The effect of reactive oxygen species on cardiomyocyte differentiation of pluripotent stem cells," Free Radical Research, vol. 52, no. 2, pp. 150-158, 2018. 